\title{
Differences in Neuroticism Between Patients with Glaucoma Who Have Discontinued Visits to Ophthalmologists and Those Who Make Regular Visits: Implications for Adherence to Topical Glaucoma Medications
}

Tadashi Nakano $\cdot$ Fumitoshi Kodaka $\cdot$ Hiroshi Tsuneoka

Received: July 1, 2016/ Published online: August 29, 2016

(C) The Author(s) 2016. This article is published with open access at Springerlink.com

\section{ABSTRACT}

Introduction: Neuroticism is a personality trait often described in individuals with glaucoma (GLC), but is not necessarily representative of the total population of patients. There is a population of patients with GLC who are invisible to clinical ophthalmologists; in other words, those who once have been diagnosed with GLC, but spontaneously stop visiting an ophthalmologist. Little is known about their neuroticism personality trait. In the present study, the authors compared the level of neuroticism between patients no longer visiting an ophthalmologist and those who continue visit them regularly.

Methods: Patients were assigned to two groups according to the duration of their last visit to an

Enhanced content To view enhanced content for this article go to http://www.medengine.com/Redeem/2FE4 F0605E30C26D.

T. Nakano $(\varangle) \cdot$ H. Tsuneoka

Department of Ophthalmology, Jikei University

School of Medicine, Tokyo, Japan

e-mail: tnakano@jikei.ac.jp

F. Kodaka

Department of Psychiatry, Jikei University School of

Medicine, Tokyo, Japan ophthalmologist: the Discontinued group included those patients who had not visited an ophthalmologist for the last 6 months, and the Regular Visitor group included those patients who continued to make regular visits to an ophthalmologist. The Japanese version of Ten Items Personality Inventory (TIPI-J), a questionnaire specifically used to assess the Big Five personality traits (extraversion, agreeableness, conscientiousness, neuroticism, and openness), was completed by patients through a dedicated website.

Results: Three-hundred and seventy-three patients with GLC were recruited. The neuroticism score from the TIPI-J in the Discontinued group was significantly lower than that in the Regular Visitor group $(7.63 \pm 2.23$ vs. $8.23 \pm 2.21$, respectively; $P=0.01)$. No significant difference was found in the other TIPI-J sub-item scores between the Discontinued and the Regular Visitor groups.

Conclusion: In this study, the authors showed that neuroticism trait in patients with GLC who have discontinued visiting their ophthalmologists was lower than that in patients who regularly visited them, on the basis of the Big Five personality traits as measured by the TIPT-J. 
Funding: Santen Pharmaceutical Co., Ltd.

Keywords: Five-factor model of personality; Glaucoma; Neuroticism; The Big Five personality traits

\section{INTRODUCTION}

Neuroticism is a personality trait often described in individuals with glaucoma (GLC), and has been suggested to be a possible cause of fluctuations in intraocular pressure [1]. Early studies have described patients with GLC as anxious, overconscientious [2], depressive, hysterical, meticulous, and perfectionists [3-6]. Recent studies using the five-factor model of personality [7], which describes human personality as a combination of extraversion, agreeableness, conscientiousness, neuroticism, and openness, have suggested that patients with GLC have a greater level of neuroticism than patients with cataracts [8], indicating that patients with GLC might have a neurotic personality.

However, such a neuroticism-featuring personality is not necessarily representative of the total population of patients with GLC. A previous study has revealed that adherence and persistence with medication in patients with GLC was lower than that for of individuals with other chronic diseases, such as diabetes, dyslipidemia, and osteoporosis [9]. In that study, over $50 \%$ of patients with GLC stopped their medications within 6 months [9]. Another study has suggested that this trend was similar regardless of the class of medication, for example, with prostaglandins, alpha-agonists, and beta-blockers [10]. This suggests that there is likely to be a population of patients suffering from GLC who discontinue regular visits an ophthalmologist and are thus are invisible to healthcare workers. As a result, little will also be known about their neuroticism personality trait.

In the present study, the authors compared the level of neuroticism between a population of patients with GLC who have discontinued their visits to ophthalmological clinics and a population who maintain their regular visits.

\section{METHODS}

\section{Patients}

Patients with glaucoma were recruited from a consumer investigator database (Macromill, Inc., Tokyo, Japan). Invitation letters were sent to eligible candidates. After the study protocol was presented at the dedicated website of Macromill, Inc., informed consent was obtained by clicking an agreement button. All enrolled patients had to have been diagnosed with GLC and they had to have received prescriptions by ophthalmologists before participation in the present study. Diagnosis, type of GLC, and prescription information was self-disclosed by the participants. This study protocol was approved by the Ethics Committee of Jikei University School of Medicine.

\section{Assessment Procedures}

All assessments were conducted through the dedicated website of Macromill, Inc. Participants were assessed on their duration of visits to ophthalmologists: $0=$ regularly visits, 1 = discontinued for less than 1 month, $2=$ discontinued for $1-3$ months, $3=$ discontinued for 3-6 months, and $4=$ discontinued for over 6 months. All participants completed the assessment procedures and were subsequently 
assigned to two groups based on the continued duration of their visits to their ophthalmologists. These groups were referred to as the Discontinued group (participants rated as 1, 2, 3, and 4) and the Regular Visitor group (those rated as 0). Thus 'discontinued' was defined as the duration (or number of days) from an appointment day when the participants were supposed to but did not visit their ophthalmologist. Stratified sampling was conducted by three age groups (40-49 years old, 50-59 years old, and 60 years old and over), as well as gender groups (male and female). The sample size was adopted from the previous study by Mabuchi et al. [8]. All patients underwent a personality evaluation using the Japanese version of the ten-item personality inventory (TIPI-J).

\section{Japanese Version of the Ten-Item Personality Inventory (TIPI-J)}

The ten-item personality inventory (TIPI) is a questionnaire specifically used to evaluate the 'Big Five' personality traits [11]. The Big Five personality traits comprise five factors of personality traits, referred to as extraversion, agreeableness, conscientiousness, neuroticism, and openness to experience [12].

TIPI can be applied to large-scale research due to the minimal number of items to be answered as compared with other authorized questionnaires, such as the revised neuroticism-extraversion-openness personality inventory (NEO PI-R), comprising 240 items, and the five-factor personality questionnaire 50 (FFPQ-50), comprising 50 items. The Japanese version of TIPI (TIPI-J) has a validity equal to the FFPQ-50 [13], indicating that the TIPI-J questionnaire specifically evaluates the Big Five personality traits. TIPI items are rated from 1 (strongly disagree) to 7 (strongly agree) by the participants. The TIPI items are as follows:

1. Extraverted, enthusiastic

2. Critical, quarrelsome

3. Dependable, self-disciplined

4. Anxious, easily upset

5. Open to new experiences, complex

6. Reserved, quiet

7. Sympathetic, warm

8. Disorganized, careless

9. Calm, emotionally stable

10. Conventional, uncreative.

\section{Data Analysis}

All data analyses were performed with R statistical environment 3.2.3 (R Development Core Team) and Prism 6.0 software (GraphPad Software Inc., San Diego, CA, USA). To evaluate each personality trait, the authors adopted the following calculation [13]: extraversion: item $1+(8-$ item 6); agreeableness: $(8-$ item 2$)+$ item 7 ; conscientiousness: item $3+(8-$ item 8$)$; neuroticism: item $4+(8$ - item 9$)$; and openness to new experiences: item $5+(8-$ item 10$)$. To evaluate the personality profile characteristics of the Discontinued group, the authors performed a two-sample $t$ test between the Discontinued and Regular Visitor groups for each sub-item score from TIPI-J. Statistical significance was set at less than $5 \%$ for each sub-item.

To assess the relationship between personality trait and the duration of non-visits, the authors performed correlation analysis between the sub-item(s) from TIPI-J that showed statistical significance by two-sample $t$-test and the frequency of non-visits to ophthalmologists in the Discontinued group. Spearman's rank correlation coefficient $(\rho)$ was used to examine the relationship between the personality trait sub-item(s) and the duration of non-visits. Statistical significance was set at less than 5\%. 


\section{RESULTS}

Invitations were sent to 494 study candidates, and 373 individuals spontaneously visited the dedicated website of Macromill, Inc. No individuals declined to participate in the study, thus 373 patients with GLC were recruited to the study. The response rate, calculated as the number of participants enrolled relative to the number of invitations sent, was $75.5 \%$.

\section{Patient Demography}

The Discontinued group consisted of 186 participants and the Regular Visitor group consisted of 187 participants. The mean age of patients in the Discontinued group was significantly higher than that of patients in the Regular Visitor group $(58.7 \pm 10.1$ years vs. $56.3 \pm 10.0$, respectively, $p=0.02$; Table 1 ). No significant difference was found in the gender ratio of the two groups ( $74.5 \%$ male vs. $50.3 \%$ male, $P=0.18$; Table 1 ). The median non-visit duration of the Discontinued group was over 6 months.

\section{Comparison of Sub-items from TIPI-J Between Discontinued and Regular Visitor Groups}

The mean neuroticism score from the TIPI-J for the Discontinued group was significantly lower than that for the Regular Visitor group $(7.63 \pm 2.23$ vs. $8.23 \pm 2.21$, respectively, $p=0.01$; Table 2 ). No significant difference was found in extraversion, agreeableness, conscientiousness, and openness to new experiences scores between the Discontinued and the Regular Visitor groups (Table 2). The scores in the Discontinued group were almost identical to those in the Regular Visitor group $\quad(7.53 \pm 2.48$ vs. $7.28 \pm 2.48$ for extraversion, $9.63 \pm 2.01$ vs. $9.74 \pm 2.02$ for agreeableness, $8.39 \pm 2.28$ vs. $8.54 \pm 2.37$ for conscientiousness, and $7.93 \pm 2.03$ vs. $7.88 \pm 2.15$ for openness to new experiences, respectively; Table 2).

\section{Relationship Between the Neuroticism Trait and Duration of Non-visits}

No significant correlation was found between the neuroticism score from TIPI-J and the

Table 1 Comparison of demography between Discontinued and Regular Visitors to ophthalmologists in patients with glaucoma

\begin{tabular}{llll}
\hline & Discontinued group & Regular Visitor group $^{P}$ value \\
\hline$N$ & 188 & 187 \\
Male gender (\%) & 74.5 & 50.3 & 0.18 \\
Age (mean \pm SD) (years) & $58.7 \pm 10.1$ & $56.3 \pm 10.0$ & 0.02 \\
Duration of non-visits to ophthalmologist (mode) & $>6$ months & N/A &
\end{tabular}

No significant difference was found in the gender ratio between the Discontinued group (those patients who have not visits an ophthalmologist for the last 6 months) and the Regular Visitors group (those patients who continue to make regular visits to ophthalmologists); a significant difference was found in the mean age of patients between the two groups

$N / A$ not applicable, $S D$ standard deviation

a Two-sample $t$-test was performed for age, and Chi square test for gender ratio between the Discontinued and Regular Visitor groups to ophthalmologists in patients with glaucoma. Statistical significance of both tests was set at less than $5 \%$ 
Table 2 Comparison of sub-item scores from the TIPI-J between the Discontinued and Regular Visitors groups

\begin{tabular}{llll}
\hline Sub-items from TIPI-J & $\begin{array}{l}\text { Discontinued } \\
(\text { Mean } \pm \text { SD })\end{array}$ & $\begin{array}{l}\text { Regular Visitor } \text { group } \\
(\text { Mean } \pm \text { SD })\end{array}$ & $\boldsymbol{P}^{\text {value }^{\mathbf{a}}}$ \\
\hline Extraversion & $7.53 \pm 2.48$ & $7.28 \pm 2.48$ & 0.32 \\
Agreeableness & $9.63 \pm 2.01$ & $9.74 \pm 2.02$ & 0.60 \\
Conscientiousness & $8.39 \pm 2.28$ & $8.54 \pm 2.37$ & 0.51 \\
Neuroticism & $7.63 \pm 2.23$ & $8.23 \pm 2.21$ & 0.01 \\
Openness to experiences & $7.93 \pm 2.03$ & $7.88 \pm 2.15$ & 0.25 \\
\hline
\end{tabular}

The Discontinued group (those patients who have not visits an ophthalmologist for the last 6 months) had a significantly higher score only in the neuroticism sub-item when compared to the Regular Visitor group (those patients who continue to make regular visits to ophthalmologists)

$S D$ standard deviation, TIPI-J Japanese version of ten items of personality inventory

a Two-sample $t$-test was performed for each sub-scale of TIPI-J. Statistical significance was set at less than 5\%

duration of non-visits to ophthalmologists in the Discontinued group ( $\rho=0.10, P=0.16)$.

\section{DISCUSSION}

In the present study, the authors have shown that the neuroticism trait, based on the Big Five personality traits as measured by the TIPI-J, was lower in the patients with GLC who discontinued their visits to ophthalmologists compared to those patients who continued to make regular visits.

\section{Comparison of Sub-items from TIPI-J Between the Discontinued and Regular Visitor Groups}

The mean neuroticism score from the TIPI-J in the Discontinued group was found to be significantly lower than that in the Regular Visitor group, while no significant difference was found in the scores from the other sub-items. Individuals with high neuroticism, or a tendency to experience negative emotions and emotional distress [14], can have somatic sensitivity [15] and worries about their health
[16]. In the current study, it is hypothesized that the Discontinued group could readily suspend their visits to ophthalmologists due to lower neuroticism than the Regular Visitor group.

\section{Relationship Between the Neuroticism Trait and the Duration of Discontinued Visits to Ophthalmologists}

No significant correlation was found between the neuroticism score from the TIPI-J and the duration of non-visits to ophthalmologists in the Discontinued group. Thus, the authors suggest that the lack of correlation is due to the fact that the range of neuroticism trait in patients with GLC decides whether they are regular visitors or not.

\section{Neuroticism as a Target for Continuation of Topical GLC Medications}

The results of the study indicate that neuroticism could be an important target for treatment of GLC. Neuroticism itself can be an auxiliary target to treat the pathophysiology of 
GLC. High neuroticism can not only be a risk for mortality in coronary heart disease [17] and a predictor of blood pressure [18], but also a possible cause of fluctuations of intraocular pressure [1]. This indicates that psychotherapeutic intervention for high neuroticism could stabilize intraocular pressure.

Neuroticism can also be a marker by which ophthalmologists change their approach to gain adherence to topical GLC medications. In the present study, low neuroticism predicts discontinuation to visit ophthalmologists, and, because individuals with high neuroticism can be sensitive to negative emotions, providing them with prognostic information from an optimistic aspect (e.g., this medication will prevent $30 \%$ of patients with GLC from going blind) might help them to regularly visit ophthalmologists. As for individuals with low neuroticism, prognostic information from a pessimistic aspect (e.g., 70\% of patients will go blind even if they receive this medication) might encourage them to regularly visit ophthalmologists.

\section{Study Limitation}

The current study has some limitations. First, stratification by age and gender was not enough to avoid sampling bias between the Discontinued and Regular Visitor groups. Due to lack of a control group, a referential neuroticism personality trait score could not be provided. Studies that include a control group are needed. Second, although the findings of the current study could have implications for the adherence of patients to topical GLC medications, the study did not directly measure adherence and persistence, meaning that the effect of the neuroticism personality trait on GLC medication needs further study. Third, as the definition of 'discontinuation' in the current study included participants who simply forgot to visit to their ophthalmologists, this could lead to a selection bias that could overestimate the duration of the discontinuation in the Discontinued group. Further studies would need to include a stricter definition for 'discontinuation.' Fourth, diagnosis, type of GLC, and prescription was self-reported by the participants, and, therefore, the accuracy of the data obtained was not independently validated by the authors.

\section{CONCLUSIONS}

In this study, the authors showed that neuroticism trait in patients with GLC who have discontinued visiting their ophthalmologists was lower than that in patients who visited them regularly, on the basis of the Big Five personality traits as measured by the TIPT-J.

\section{ACKNOWLEDGMENTS}

We thank Chie Shimojo, M.A., for providing information about the evaluation of the Big Five personality traits. This study was supported in part by a fund from Santen Pharmaceutical Co., Ltd., Osaka, Japan. The article processing charges for this publication were funded by the authors. All authors had full access to all of the data in this study and take complete responsibility for the integrity of the data and accuracy of the data analysis. All named authors meet the International committee of medical Journal Editors (ICMJE) criteria for authorship for this manuscript, take responsibility for the integrity of the work as a whole, and have given final approval for the version to be published.

Disclosures. Tadashi Nakano received consultant fees from Topcon Corporation and lecture fees from Alcon Japan, Ltd., Santen 
Pharmaceutical Co., Ltd., Otsuka Pharmaceutical Co., Ltd., R-Tech Ueno, Ltd., Senju Pharmaceutical Co., Ltd., Carl Zeiss Co., Ltd., Kaken Pharmaceutical Co., Ltd., MSD K.K., and Pfizer Japan, Inc. Fumitoshi Kodaka has nothing to disclose. Hiroshi Tsuneoka received consultant fees from Santen Pharmaceutical Co., Ltd., Otsuka Pharmaceutical Co., Ltd., MSD K.K., Senju Pharmaceutical Co., Ltd., and AMO Japan Co., Ltd.

Compliance with Ethics Guidelines. This study protocol was approved by the Ethics Committee of Jikei University School of Medicine. After the study protocol was presented at the dedicated website of Macromill, Inc., informed consent was obtained from participants by clicking an agreement button.

Open Access. This article is distributed under the terms of the Creative Commons Attribution-NonCommercial 4.0 International License (http://creativecommons.org/licenses/ by-nc/4.0/), which permits any noncommercial use, distribution, and reproduction in any medium, provided you give appropriate credit to the original author(s) and the source, provide a link to the Creative Commons license, and indicate if changes were made.

\section{REFERENCES}

1. Berger AS, Zimet CN. Personality features of patients with primary glaucoma. A medico psychosocial exploration. Psychosom Med. 1959;21:389-96.

2. Miller SJ. Symptomatology of congestive and simple glaucoma; a study in contrast. Br Med J. 1952;1(4756):456-61.

3. Hibbeler HL. Personality patterns of white adults with primary glaucoma. Am J Ophthalmol. 1947;30(2):181-6.
4. Zimet CN, Berger AS. Emotional factors in primary glaucoma: an evaluation of psychological test data. Psychosom Med. 1960;22:391-9.

5. Labib MA, Abboud I, Shaheen O, el-Rifai M. Primary glaucoma profile on the Minnesota multiphasic personality inventory. Bull Ophthalmol Soc Egypt. 1970;63(67):127-37.

6. Shaheen O, Labib MA, Abboud I, el-Rifai M. Some ophthalmological factors affecting the personality patterns in primary glaucoma patients as determined by the Minnesota multiphasic personality inventory. Bull Ophthalmol Soc Egypt. 1970;63(67):139-40.

7. McCrae RR. The five-factor model and its assessment in clinical settings. J Pers Assess. 1991;57(3):399-414.

8. Mabuchi F, Yoshimura K, Kashiwagi K, Shioe K, Kanba S, Iijima $\mathrm{H}$, et al. Personality assessment based on the five-factor model of personality structure in patients with primary open-angle glaucoma. Jpn J Ophthalmol. 2005;49(1):31-5.

9. Yeaw J, Benner JS, Walt JG, Sian S, Smith DB. Comparing adherence and persistence across 6 chronic medication classes. J Manag Care Pharm. 2009;15(9):728-40.

10. Nordstrom BL, Friedman DS, Mozaffari E, Quigley HA, Walker AM. Persistence and adherence with topical glaucoma therapy. Am J Ophthalmol. 2005;140(4):598-606.

11. Gosling SD, Rentfrow PJ, Swann WB Jr. A very brief measure of the big-five personality domains. J Res Pers. 2003;37:504-28.

12. Goldberg LR. An alternative "description of personality": the big-five factor structure. J Pers Soc Psychol. 1990;59(6):1216-29.

13. Oshio A, Abe S, Cutrone P. Development, reliability, and validity of the Japanese version of Ten Item Personality Inventory (TIPI-J). Jpn J Pers. 2012;21(1):40-52.

14. De Jong GM, van Sonderen E, Emmelkamp PM. A comprehensive model of stress. The roles of experienced stress and neuroticism in explaining the stress-distress relationship. Psychother Psychosom. 1999;68(6):290-8.

15. Costa PT Jr, McCrae RR. Neuroticism, somatic complaints, and disease: is the bark worse than the bite? J Pers. 1987;55(2):299-316.

16. Friedman HS. Long-term relations of personality and health: dynamisms, mechanisms, tropisms. J Pers. 2000;68(6):1089-107. 
17. Jokela M, Pulkki-Raback L, Elovainio M, Kivimaki M. Personality traits as risk factors for stroke and coronary heart disease mortality: pooled analysis of three cohort studies. J Behav Med. 2014;37(5):881-9.
18. Turiano NA, Pitzer L, Armour C, Karlamangla A, Ryff CD, Mroczek DK. Personality trait level and change as predictors of health outcomes: findings from a national study of Americans (MIDUS). J Gerontol B Psychol Sci Soc Sci. 2012;67(1):4-12. 